\title{
Research on Economics of Mixed Energy Storage for Smoothing Wind Power Fluctuation with Consideration of Confidence Level
}

\author{
Xintao XIE ${ }^{1}$, Hao LI,a, Liqiang $\mathrm{PAN}^{3}$, Shuai $\mathrm{XIAO}^{1}$, Mengjiao $\mathrm{LI}^{1}$ and Zhenyu WU² \\ ${ }^{1}$ State Grid Hunan Electric Power Company Economic and Technological Research Institute, Changsha 410004, China \\ ${ }^{2}$ Changsha University of Science \& Technology, Changsha 410004, China \\ ${ }^{3}$ State Grid Hunan Electric Power Corporation Limited, Changsha 410004,China
}

\begin{abstract}
This paper proposes a research method for mitigating wind power fluctuations in a hybrid energy storage system considering the confidence level of wind power volatility. First, the typical daily wind power output is subjected to Fourier spectrum analysis to determine the frequency range of wind power output, and combined with low-pass filtering to find the optimal cutoff frequency, for obtaining energy storage reference power; Then determining the optimal energy storage reference power by comparing the energy storage reference powers solved at different confidence levels. Finally, empirical mode decomposition (EMD) is used to decompose the stored energy into a series of intrinsic mode functions(IMFs), demarcation of the energy storage reference power with minimum aliasing in the instantaneous frequency-time curve, the high frequency part is configured by power type energy storage, and the low frequency part is configured by energy type energy storage. The simulation of the wind farms in a certain area of Hunan Province is carried out. The result shows that proper reduction of confidence level can reduce energy storage power and capacity.
\end{abstract}

\section{Introduction}

Since the beginning of the 21 st century, energy issues have become a worldwide problem. As more and more new energy sources are discovered, the problem of traditional energy depletion is expected to be solved. However, due to the intermittent and random nature of its output, new energy power generation is difficult to be directly utilized by the power grid. Adding an energy storage device can solve this problem well ${ }^{[1,2]}$.

Driven by the rapid development of scientific and technological innovation, energy storage technology has also been rapidly improved, and the application of energy storage has been more and more recognized. The resulting energy storage and participation in the optimal allocation of new energy sources is also increasing. At present, there are many methods such as the sliding average method, the low-pass filtering method and the empirical mode decomposition method for smoothing wind power. The participation of energy storage systems can make the power system of new energy sources such as wind power run more stably, but how to configure its power and capacity becomes a problem that needs to be studied. At present, the main capacity allocation and optimization methods include the difference supplementary method, the fluctuation flat analysis method and the economic characteristic optimization method (that is, the economic evaluation method). According to the related characteristics of time and space of wind power and energy storage system and the linkage of wind and fire

a Corresponding author: 2992906560@qq.com storage, the paper [3] constructed the multi-dimensional transport characteristics of power transmission and energy space-time and the periodic cycle and power of energy storage system. In [4], an electromechanical static model considering flow energy storage is established, and then the energy storage optimization configuration of the wind energy integration system is realized by simulation calculation. Reference [5] proposed a wind power filtering method that uses hybrid energy storage system (HESS) to mitigate short-term and long-term fluctuations, and optimizes the configuration of hybrid energy storage based on wavelet transform method. Reference [6] discusses the implementation of battery energy storage (BESS) in the first and second frequency modulation of the power grid, and proposes a BESS optimization configuration method with the same frequency modulation capability as the thermal power unit.

\section{Wind power fluctuation smoothing method considering wind power volatility confidence level}

The traditional wind power volatility is that the wind power output curve volatility is all less than the set value, and for different confidence levels, the wind power volatility is less than the power volatility set value with different probability. The probability is defined as follows: 


$$
p=\frac{N_{x}}{N} \times 100 \%
$$

Where: $\mathrm{p}$ is the confidence level value, $\mathrm{Nx}$ is the number of periods in which the fluctuation rate is less than the set value in the period of the period, and $\mathrm{N}$ is the number of periods in the period of the period.

\section{Energy storage optimization configuration method considering confidence level}

\subsection{The energy storage reference power considering the confidence level}

The low frequency part of the wind power output is directly supplied to the grid, and the high frequency part is absorbed or released by the energy storage. The energy storage power at time $\mathrm{k}$ is expressed as:

$$
P_{E 0(k)}=P_{f(k)}-P_{\text {line }(k)}=\frac{\tau}{\Delta t+\tau}\left(P_{f(k)}-P_{\text {line }(k-1)}\right)
$$

Where: $P_{E 0(k)}$ is the power absorbed or released by the stored energy at time $\mathrm{k}$.

(2) is the equation for the energy storage power, the energy storage discharge power is negative, the charging power is positive, and the energy storage power at different confidence levels is solved by changing the time constant $\tau$, for a certain volatility setting Value, the lower the confidence level, the smaller the time constant. In view of (2), the larger the value of $\tau$ is, the closer $P_{\text {line }(k)}$ is to $P_{\text {line }(k-1)}$, the better the power leveling effect, but the greater difference between the $P_{\text {line }(k)}$ and $P_{f(k)}$, which will lead to an increase in energy storage costs. Therefore, how to choose the appropriate $\tau$ becomes the key.

\subsection{Hybrid energy storage system power allocation}

In this paper, the energy storage requirements are decomposed by EMD, and the distribution is based on the most modal aliasing of adjacent IMFs in the frequency-time curve. The assigned high and low frequency signal components are respectively used as reference powers for power type and energy type energy storage.

\subsection{Calculation of charge and discharge}

The charge and discharge power of the stored energy is obtained from the energy storage reference power, and the expression is:

$$
E[k]=\sum_{0}^{N} P_{E}^{\prime}[k] \cdot \frac{T_{S}}{3600}, k=0,1,2,3 \ldots N
$$

Where Dividing by 3600 is to convert the time into hours, and the obtained unit of electricity is $\mathrm{kWh}$.

\subsection{Rated capacity calculation}

The residual energy change of the energy storage system can be expressed by State of Charge (SOC), which is calculated as:

$$
S=S_{0}+\frac{E[n]}{E_{N}}
$$

Where: $\mathrm{S}$ is the real-time state of charge; $\mathrm{S}_{0}$ is the initial state of charge; $E_{N}$ is the rated capacity of energy storage. When charging, $\mathrm{E}[\mathrm{n}]$ is positive, residual energy increases, and SOC increases; when discharging, $E[n]$ is negative, residual energy decreases, and SOC decreases.

The initial SOC and energy of energy storage should be able to meet: Under a certain SOC, the current time of the energy storage system can not be less than the SOC minimum limit, and the current capacity of the energy storage system cannot exceed the maximum SOC limit. According to formula (3), the maximum positive energy fluctuation value can be obtained as $\max \{E[n]\}$, and the maximum negative energy fluctuation value is $\min \{\mathrm{E}[\mathrm{n}]\}$.

Set the maximum and minimum allowable values of the state of charge of the energy storage system to Smax and Smin, respectively:

$$
\begin{gathered}
S_{\max }-S_{0} \leq \frac{\max \{E[n]\}}{E_{N}} \\
S_{0}-S_{\min } \geq \frac{\min \{E[n]\}}{E_{N}}
\end{gathered}
$$

The solution can be:

$$
E_{N}=\frac{\max \{E[n]\}+|\min \{E[n]\}|}{S_{\max }-S_{\min }}
$$

\subsection{Initial state determination}

The initial state of charge can be obtained by the equalization of equations (5) and (6):

$$
S_{0}=\frac{\min \{E[n]\} S_{\max }-\max \{E[n]\} S_{\min }}{\min \{E[n]\}-\max \{E[n]\}}
$$

Therefore, the maximum positive energy fluctuation value and the maximum negative energy fluctuation value of the stored energy are obtained by the energy storage reference power value. After the maximum and minimum range of the SOC is given, the minimum rated capacity of the energy storage can be obtained, and the energy storage can be obtained Initial state. As long as the energy storage is adjusted to the initial value state, the charge and discharge requirements in the entire cycle can be satisfied.

\section{Case analysis}

The data used in the simulation is the data of a wind farm in Hunan. The construction scale of A wind farm is 50MW. 
25 wind turbines with a single unit capacity of $2 \mathrm{MW}$ are installed. The wind power output data of 96 points on the day of 2016 is analyzed. The volatility standard is specified in this paper. The volatility for 30 minutes is less than $10 \%$.

The maximum volatility reached 0.59 before the smoothing wind. The key to low-pass filtering is to find the appropriate time constant $\tau$. In this paper, the optimal time constant $\tau$ is found by performing Fourier spectrum analysis on typical daily wind power output. The typical daily spectral curve is shown in Fig. 1.

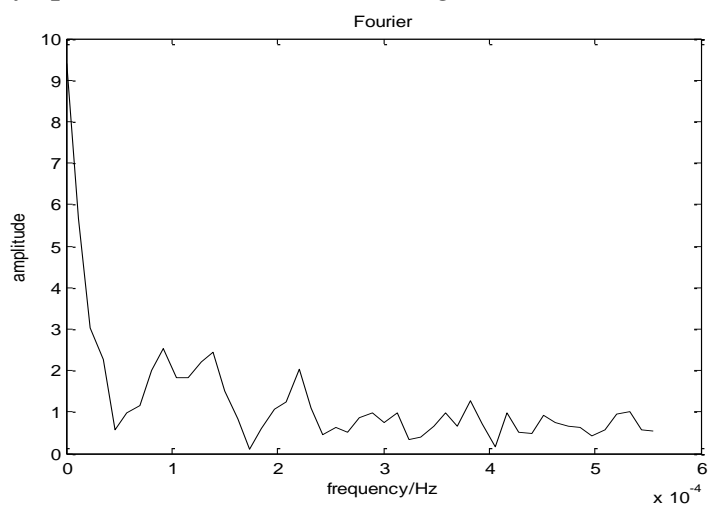

Figure. 1 A wind farm typical wind power output spectrum

It can be seen from the figure that the frequency variation of the typical daily wind power output of the A wind farm is mainly concentrated in $1.16 \times 10^{-5} \sim 5.67 \times 10^{-4} \mathrm{~Hz}$. The amplitude of the low frequency part is higher than that of the high frequency part, and when the frequency reaches a certain level, the attenuation is zero.

According to the typical sunrise force spectrum of wind farms, several sets of time constants are selected from low frequency to high frequency. The wind power output suppression effect is obviously different under different time constants. Figure 2 is the waveform diagram after different time constants are flattened.

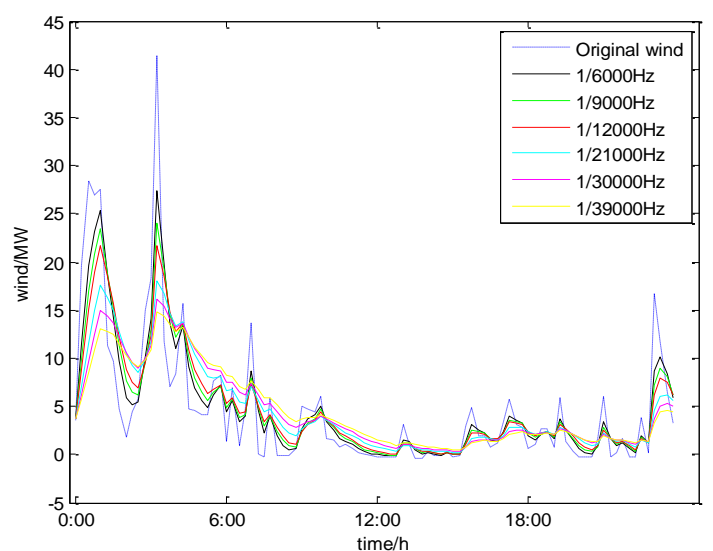

Figure. 2 Different cut-off frequencies into the network power

It can be seen from Fig. 2 that the power of the tie line at different cutoff frequencies is different. The lower the cutoff frequency, that is, the larger the time constant, the better the fluctuation suppression effect. When the cutoff frequency is $1 / 39000 \mathrm{~Hz}$, the wind power fluctuation rate Meet the requirements.
According to the energy storage reference power under different confidence levels, for the capacity configuration of the hybrid energy storage. For example the confidence level is $100 \%$. At this time, the reference energy of the energy storage is $26.65 \mathrm{MW}$, and the reference power curve is EMD decomposed. The waveform is shown in Figure 3. Figure 4 shows the IMF instantaneous frequency curve. It can be seen from the figure that IMF3 and IMF4 have almost no overlap. Therefore, IMF1 to IMF3 are divided into high-frequency part energy storage reference power, and IMF4 to IMF6 are low-frequency part energy storage reference power.
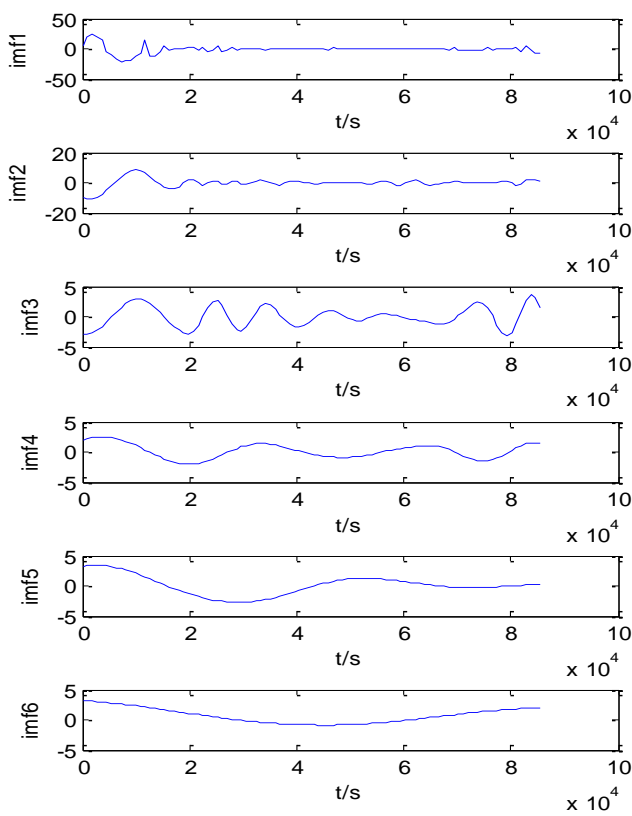

Figure. 3 All IMFs curves

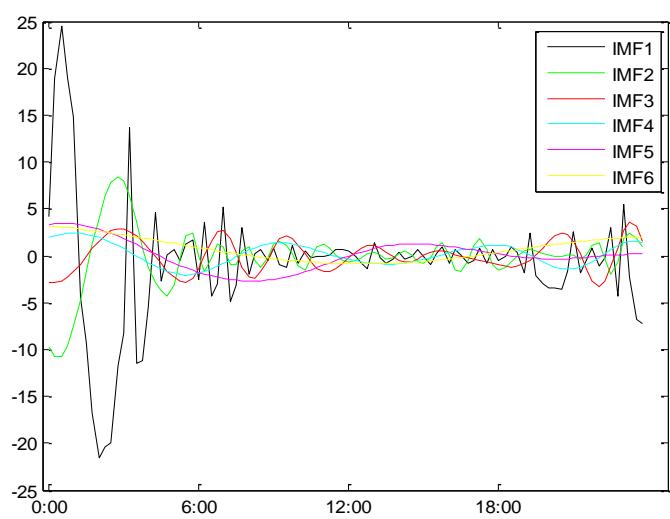

Figure. 4 IMF instantaneous frequency curve

The energy storage reference power is divided into two parts, a high frequency and a low frequency, and a power type super capacitor and an energy type lithium ion battery are respectively used as an energy storage power source for configuration. The power and capacity at different confidence levels are shown in Table 1.

Table 1 Energy power, capacity, and initial SOC at different confidence level

Confidence level Power Capacity Initial




\begin{tabular}{llccc}
\hline & & $(\mathrm{MW})$ & $(\mathrm{MWh})$ & SOC \\
\hline \multirow{2}{*}{$8.38 \%$} & Energy type & 1.61 & 8.30 & 0.15 \\
& Power type & 13.25 & 16.83 & 0.65 \\
\multirow{2}{*}{$87.50 \%$} & Energy type & 3.92 & 12.18 & 0.15 \\
& Power type & 14.51 & 25.29 & 0.65 \\
\multirow{2}{*}{$90.63 \%$} & Energy type & 2.21 & 13.27 & 0.15 \\
& Power type & 17.96 & 26.57 & 0.65 \\
\multirow{2}{*}{$93.75 \%$} & Energy type & 5.97 & 26.95 & 0.15 \\
& Power type & 18.77 & 36.05 & 0.73 \\
$96.88 \%$ & Energy type & 7.09 & 28.86 & 0.15 \\
& Power type & 20.84 & 40.46 & 0.74 \\
$100 \%$ & Energy type & 7.95 & 32.85 & 0.15 \\
& Power type & 21.57 & 41.62 & 0.73 \\
\hline
\end{tabular}

It can be seen from Table 1 that the initial SOC of the energy storage system does not change at different confidence levels, both being 0.15 . The initial SOC of the power storage system also did not change much. The confidence level was 0.65 at $84.38 \%, 87.50 \%$, and $90.63 \%$; the confidence level was 0.73 or 0.74 at $93.75 \%, 96.88 \%$, and $100 \%$. The energy storage capacity and capacity are basically increased with the increase of the confidence level. The power of the energy storage system is reduced only when the confidence level changes from $87.50 \%$ to $90.63 \%$

\section{Conclusion}

This paper proposes a new smoothing wind power method based on low-pass filtering, and draws the following conclusions: 1) Selecting the cutoff frequency through the amplitude-frequency characteristic curve enables the calculation result to be more accurate under the same energy storage configuration. 2) Properly reducing the confidence level can reduce the capacity and power of the energy storage configuration. 3) Considering the power compensation, the initial state of charge of the energy storage is the same every day, preventing overcharging or overdischarging of the energy storage.

\section{References}

1. Tang Xisheng, Deng Wei, Li Ningning, et al: Control technologies of micro-grid operation based on energy storage. Electric Power Automation Equipment 2012, 32(3): 99-103.

2. Sang Bingyu, Wang Deshun, Yang Bo, et al: Optimized Energy Storage Configuration for Smoothing New Energy Output Volatility, Proceedings of the CSEE. 2014,34(22):3700-3706.

3. Guo Zhuangzhi, Hong Junjie, Meng Anbo, et al: Multi-indicator optimal allocation strategy for large-scale grid-connected wind energy storage capacity based on multi-dimensional dynamic coordination, Electric Power Automation Equipment, 2016,36(02):161-168.

4. Xiang Yuzheng; RONG Jia, et al.Energy storage capacity configuration for large-scale wind power accessed to saturated system. 2016 China
International Conference on Electricity Distribution (CICED),2016,1-5.

5. Quanyuan Jiang; Haisheng Hong.Wavelet-Based Capacity Configuration and Coordinated Control of Hybrid Energy Storage System for Smoothing Out Wind Power Fluctuations,IEEE Transactionson Power Systems, 2013,28(2),1363 - 1372.

6. Ding Dong, Yang Shuili, Li Jianli, et al, Energy Storage Science and Technology,2014, 3(04): 302-307. 